\title{
Time scale, objectivity and irreversibility in quantum mechanics
}

\author{
L. Lanz"] and O. Melsheimer"
}

\section{Introduction}

For the physical interpretation of the formalism of quantum mechanics (QM) a classical world of sources and detectors of microsystems must be invoked in order to get rid of the so-called E.R.P. paradox and the subjectivity within the measuring process. Substituting invocation with high mathematical effort, Ludwig obtained QM as the description of a microphysical interaction channel between macrosystems and showed that QM must be enriched by the mathematical tools that are now called POV measures, operations and instruments (for a more recent overview see (Ludwig, 1983)). It is very remarkable that these concepts also arose in more accurate descriptions of the measuring process and more profound thinking about the statistical structure of QM (Davies, 1976), (Holevo, 1982) and (Kraus, 1983). However the problem remains to reconcile the classical phenomenological description of systems with the quantum mechanical description of their microphysical structure. Our aim is to reconsider this problem taking in an explicit way isolated systems as basic elements of reality and looking at QM, already in its field formulation, as the basic theory of finite isolated systems; only afterwards one arrives at particles when the peculiarities of finite systems, like boundary conditions, are negligible: in this philosophy the thermodynamic limit has just a reversed role, it is not used in order to reveal the classical behavior of macrosystems, but is necessary to attain local covariance and universality of the theory. All the difficulties related to quantum mechanical inseparability, enter now in the very concept of isolated system: our point of view implies a weakening of the idea of an absolutely isolated system. Isolation is relative to a suitable set of slow variables, whose dynamics restricted to expectations, as phenomenology indicates, has only a restricted memory of the previous values of these expectations.

So to achieve isolation one has to establish a suitable time scale, choose variables with expectations having a typical variation time of this scale, prepare the system inside some confined space region during a suitable time interval, controlling and measuring the relevant variables inside a suitable preparation time interval; we have to explicitly introduce in the formalism the fact that restricting to suitable variables and using some effective description of quasilocal field interaction, the too remote history of the system

\footnotetext{
${ }^{1}$ Dipartimento di Fisica dell'Università di Milano and Istituto Nazionale di Fisica Nucleare, Sezione di Milano, Via Celoria 16, I-20133, Milan, Italy. E-mail: lanz@mi.infn.it

${ }^{2}$ Fachbereich Physik Philipps-Universität, Renthof 7,D-35032 Marburg, Germany. E-mail: melsheim@mailer.uni-marburg.de
} 
has to be neglected. On the contrary if one pretends to describe the local behavior with completely sharp time specification one can expect that the full history of the whole universe would be involved.

By taking finite isolated systems, instead of particles as the main subjects, the typical ultraviolet and infrared problem of quantum field theory is absorbed inside the non universal features of the description. The opening to irreversibility entailed by this point of view, will be discussed in Section 3 to settle problems arising in the classical description of a macrosystem; such a description is given in Section 2, essentially by a suitable reconsideration of Zubarev's approach to non equilibrium thermodynamics. As an example Boltzmann description of a dilute gas is discussed in Section 4.

\section{Description of a finite isolated system}

The microphysical structure is described by a set of interacting quantum Schrödinger fields. Here we consider the simplest model: one interacting quantum Schrödinger field (QSF) $\hat{\psi}_{n c}(\mathbf{x})$ (not yet confined), to which the following local Hamiltonian density is associated:

$$
\begin{gathered}
\hat{e}_{n c}(\mathbf{x})=\frac{\hbar^{2}}{2 m} \nabla \hat{\psi}_{n c}^{\dagger}(\mathbf{x}) \cdot \nabla \hat{\psi}_{n c}(\mathbf{x})+ \\
+\frac{1}{2} \int d^{3} \mathbf{r} \hat{\psi}_{n c}^{\dagger}(\mathbf{x}-\mathbf{r} / \mathbf{2}) \hat{\psi}_{n c}^{\dagger}(\mathbf{x}+\mathbf{r} / \mathbf{2}) V(|\mathbf{r}|) \hat{\psi}_{n c}(\mathbf{x}+\mathbf{r} / \mathbf{2}) \hat{\psi}_{n c}(\mathbf{x}-\mathbf{r} / \mathbf{2}) \\
{\left[\hat{\psi}(\mathbf{x}), \hat{\psi}^{\dagger}\left(\mathbf{x}^{\prime}\right)\right]_{ \pm}=\delta\left(\mathbf{x}-\mathbf{x}^{\prime}\right) .}
\end{gathered}
$$

The basic local dynamical variable of this model

$$
\hat{\psi}_{n c}(\mathbf{x}, t)=e^{\frac{i}{\hbar} H_{n c} t} \hat{\psi}_{n c}(\mathbf{x}) e^{-\frac{i}{\hbar} H_{n c} t} \quad, \quad \hat{H}=\int_{\omega} d^{3} \mathbf{x} \hat{e}_{n c}(\mathbf{x})
$$

satisfies the Schrödinger field equation

$$
\begin{aligned}
& i \hbar \frac{\partial \hat{\psi}_{n c}(\mathbf{x}, t)}{\partial t}=-\frac{\hbar^{2}}{2 m} \Delta \hat{\psi}_{n c}(\mathbf{x}, t)+ \\
& \quad+\int d^{3} \mathbf{y} \hat{\psi}_{n c}^{\dagger}(\mathbf{y}, t) V(|\mathbf{x}-\mathbf{y}|) \hat{\psi}_{n c}(\mathbf{y}, t) \hat{\psi}_{n c}(\mathbf{x}, t)
\end{aligned}
$$

As it is well known and will appear in the results, choice (2.1) means in usual language, Nbody system of structureless molecules interacting via the two body potential $V\left|\mathbf{x}_{1}-\mathbf{x}_{2}\right|$ ). The field equation (2.3) that accounts for covariance under Galilei transformations, has for the massive continuum we are describing a similar role that Maxwell equations have for electromagnetism, however, due to the irrelevance of self-interaction in the electromagnetic case, the classical field theory plays in the latter case a much more extended role. 
Confinement of the system inside a region $\omega \subset \mathbf{R}^{3}$ is obtained expanding $\hat{\psi}(\mathbf{x})$ on the normal modes $u_{f}(\mathbf{x})$ of the system, where

$$
-\frac{\hbar^{2}}{2 m} \Delta u_{f}(\mathbf{x})=E_{f} u_{f}(\mathbf{x}) \quad u_{f}(\mathbf{x})=0 \quad \mathbf{x} \in \partial \omega
$$

setting

$$
\hat{\psi}(\mathbf{x})=\sum_{f} u_{f}(\mathbf{x}) \hat{a}_{f} \quad\left[\hat{a}_{f}, \hat{a}_{g}^{\dagger}\right]_{ \pm}=\delta_{f g}
$$

We replace $\hat{\psi}_{n c}(\mathbf{x})$ by $\hat{\psi}(\mathbf{x})$ for $\mathbf{x} \in \omega$ and by 0 for $\mathbf{x} \notin \omega$, in the energy density $\hat{e}(\mathbf{x})$ and in all relevant expressions built with the field operators. In this way peculiar confinement is superposed to the quasilocal universal (within the limits related to the effective potential $V(|\mathbf{r}|))$ behavior.

Our aim is not at all a full description of the finite isolated system, but to give a description of it having negligible correlations with the environment; this description is related to suitable slow variables, linked to the fundamental constants of motion of the system: mass and energy.

The densities generating these observables are the relevant variables; as phenomenology indicates there are two meaningful descriptions:

A: the hydrodynamic one based on energy density and mass density,

B: the kinetic one based on energy density and phase-space density;

the energy density is given by equation (2.1) with $\hat{\psi}_{n c}(\mathbf{x})$ replaced by $\hat{\psi}(\mathbf{x})$, the mass density is given by

$$
\hat{m}(\mathbf{x})=m \hat{\psi}^{\dagger}(\mathbf{x}) \hat{\psi}(\mathbf{x})
$$

the phase-space density is given by:

$$
\hat{f}(\mathbf{x}, \mathbf{p})=\sum_{h k} \hat{a}_{h}^{\dagger}<u_{h}\left|\hat{F}^{(1)}(\mathbf{x}, \mathbf{p})\right| u_{k}>\hat{a}_{k}
$$

$\hat{F}^{(1)}(\mathbf{x}, \mathbf{p})$ being the density of a POV measure for the joint position-momentum measurement in one-particle quantum mechanics (Lanz, Melsheimer and Wacker, 1985).

The first step towards a classical description is the axiomatic introduction of a velocity field of the continuum, so that the former observables can be referred to a local rest frame: denoting by an index ${ }^{(0)}$ these observables, one has:

$$
\begin{gathered}
\hat{e}^{(0)}(\mathbf{x})=\frac{1}{2 m}(i \hbar \nabla-m \mathbf{v}(\mathbf{x}, t)) \hat{\psi}^{\dagger}(\mathbf{x}) \cdot(-i \hbar \nabla-m \mathbf{v}(\mathbf{x}, t)) \hat{\psi}(\mathbf{x})+ \\
+\frac{1}{2} \int d^{3} \mathbf{r} \hat{\psi}^{\dagger}\left(\mathbf{x}-\frac{\mathbf{r}}{2}\right) \hat{\psi}^{\dagger}\left(\mathbf{x}+\frac{\mathbf{r}}{2}\right) V(|\mathbf{r}|) \hat{\psi}\left(\mathbf{x}+\frac{\mathbf{r}}{2}\right) \hat{\psi}\left(\mathbf{x}-\frac{\mathbf{r}}{2}\right) \\
\hat{m}^{(0)}(\mathbf{x})=\hat{m}(\mathbf{x}) \\
\hat{f}^{(0)}(\mathbf{x}, \mathbf{p})=\hat{f}(\mathbf{x}, \mathbf{p}-m \mathbf{v}(\mathbf{x}, t))
\end{gathered}
$$


The introduction of this external classical field allows to compensate a gauge transformation of the field $\hat{\psi}(\mathbf{x}) \rightarrow \hat{\psi}(\mathbf{x}) e^{\frac{i}{\hbar} \Lambda(\mathbf{x})}$ with a transformation $\mathbf{v}(\mathbf{x}, t) \rightarrow \mathbf{v}(\mathbf{x}, t)-1 / m \nabla \Lambda(\mathbf{x}, t)$ of the external parameter. $\mathbf{v}(\mathbf{x}, t)$ is linked to the expectation at time $t$ of the momentum density of the system:

$$
\begin{array}{r}
\hat{\mathbf{p}}(\mathbf{x})=\frac{1}{2}\left\{\left[(i \hbar \nabla-m \mathbf{v}(\mathbf{x}, t)) \hat{\psi}^{\dagger}(\mathbf{x})\right] \hat{\psi}(\mathbf{x})-\right. \\
\left.\hat{\psi}^{\dagger}(\mathbf{x})(i \hbar \nabla+m \mathbf{v}(\mathbf{x}, t)) \hat{\psi}(\mathbf{x})\right\}
\end{array}
$$

by the relation:

$$
\langle\hat{\mathbf{p}}(\mathbf{x})\rangle_{t}=\langle\hat{m}(\mathbf{x})\rangle_{t} \cdot \mathbf{v}(\mathbf{x}, t)
$$

or equivalently

$$
\left\langle\hat{\mathbf{p}}^{(0)}(\mathbf{x})\right\rangle_{t}=0
$$

The other classical state parameters are linked to the expectations $\left\langle\hat{e}^{(0)}(\mathbf{x})\right\rangle_{t}$ and $\left\langle\hat{m}^{(0)}(\mathbf{x})\right\rangle_{t}$ (or $\left\langle\hat{f}^{(0)}(\mathbf{x}, \mathbf{p})\right\rangle_{t}$ in the kinetic case), as they enter into the structure of the most unbiased statistical operator, giving these assigned expectations. Such operator is characterized by the conditions:

$$
\begin{gathered}
\left\langle\hat{e}^{(0)}(\mathbf{x}\rangle_{t}=\operatorname{Tr}\left(\hat{\mathrm{e}}^{(0)}(\mathbf{x}) \hat{\rho}\right), \quad\left\langle\hat{\mathrm{m}}^{(0)}(\mathbf{x})\right\rangle_{\mathrm{t}}=\operatorname{Tr}\left(\hat{\mathrm{m}}^{(0)}(\mathbf{x}) \hat{\rho}\right)\right. \\
0=\operatorname{Tr}\left(\hat{\mathbf{p}}^{(0)}(\mathbf{x}) \hat{\rho}\right)
\end{gathered}
$$

$S(\hat{\rho})=-\operatorname{Tr}(\hat{\rho} \log \hat{\rho})$ being maximal. The solution of this problem of conditioned maximality is a generalized Gibbs state

$$
\hat{w}[\beta(t), \mu(t), \mathbf{v}(t)]=\frac{e^{-\int_{\omega} d^{3} \mathbf{x} \beta(\mathbf{x}, t)\left[\hat{e}^{(0)}(\mathbf{x})-\mu(\mathbf{x}, t) \hat{m}^{(0)}(\mathbf{x})\right]}}{\operatorname{Tr} e^{-\int_{\omega} d^{3} \mathbf{x} \beta(\mathbf{x}, t)\left[\hat{e}^{(0)}(\mathbf{x})-\mu(\mathbf{x}, t) \hat{m}^{(0)}(\mathbf{x})\right]}},
$$

where the fields $\beta(\mathbf{x}, t), \mu(\mathbf{x}, t)$ are determined by the equations:

$$
\begin{aligned}
\left\langle\hat{e}^{(0)}(\mathbf{x})\right\rangle_{t} & =\operatorname{Tr}\left(\hat{\mathrm{e}}^{(0)}(\mathbf{x}) \hat{\mathrm{w}}[\beta(\mathrm{t}), \mu(\mathrm{t}), \mathbf{v}(\mathrm{t})]\right) \\
\left\langle\hat{m}^{(0)}(\mathbf{x})\right\rangle_{t} & =\operatorname{Tr}\left(\hat{\mathrm{m}}^{(0)}(\mathbf{x}) \hat{\mathrm{w}}[\beta(\mathrm{t}), \mu(\mathrm{t}), \mathbf{v}(\mathrm{t})]\right)
\end{aligned}
$$

in terms of the assigned expectations $\left.\left\langle\hat{e}^{(0)}(\mathbf{x})\right\rangle_{t}, \hat{m}^{(0)}(\mathbf{x})\right\rangle_{t}$ and the given field $\mathbf{v}(\mathbf{x}, t)$ (for simplicity the dependence of $\hat{e}^{(0)}(\mathbf{x})$ on this field has not been made explicit). In the kinetic case $\hat{m}^{(0)} \rightarrow \hat{f}^{(0)}$ and $\mu(\mathbf{x}, t) \rightarrow \mu(\mathbf{x}, \mathbf{p}, t)$. The state function

$$
S(\beta(t), \mu(t), \mathbf{v}(t))=\operatorname{Tr}(\hat{\mathrm{w}}[\beta(\mathrm{t}), \mu(\mathrm{t}), \mathbf{v}(\mathrm{t})] \log \hat{\mathrm{w}}[\beta(\mathrm{t}), \mu(\mathrm{t}), \mathbf{v}(\mathrm{t})])
$$

is the thermodynamic entropy of the system. Let us take at the moment for granted that the dynamics of the system is given by the unitary evolution, generated by the Hamiltonian:

$$
\hat{H}=\int_{\omega} d^{3} \mathbf{x} \hat{e}(\mathbf{x})
$$


then the main point is to give at some time $T$ the initial statistical operator $\hat{\rho}_{T}$. Let us investigate what happens if one takes:

$$
\hat{\rho}_{T}=\hat{w}[\beta(T), \mu(T), \mathbf{v}(T)]
$$

then by a straightforward calculation (Robin, 1990), the statistical operator of the system is:

$$
\hat{\rho}_{t}=e^{-\frac{i}{\hbar} \hat{H}(t-T)} \hat{\rho}_{T} e^{\frac{i}{\hbar} \hat{H}(t-T)}
$$

which can be written in the following form:

$$
\hat{\rho}_{t}=\frac{e^{-\left\langle\beta(t) \cdot \hat{e}^{(0)}\right\rangle+\left\langle[\beta(t) \mu(t)] \cdot \hat{m}^{(0)}\right\rangle+\int_{T}^{t} d \tau \hat{S}_{t}[\beta(\tau), \mu(\tau), \mathbf{v}(\tau)]}}{\operatorname{Tr}^{-\left\langle\beta(\mathrm{t}) \cdot \hat{e}^{(0)}\right\rangle+\left\langle[\beta(\mathrm{t}) \mu(\mathrm{t})] \cdot \hat{\mathrm{m}}^{(0)}\right\rangle+\int_{\mathrm{T}}^{\mathrm{t}} \mathrm{d} \tau \hat{\mathrm{S}}_{\mathrm{t}}[\beta(\tau), \mu(\tau), \mathbf{v}(\tau)]}}
$$

The first two terms in the exponent are a more compact notation to represent the typical exponent of a Gibbs state with parameters $\beta(t), \mu(t), \mathbf{v}(t)$ (the latter is implicit inside $\left.\hat{e}^{(0)}\right)$ referring to time $t$, e.g.:

$$
\left\langle\beta(t) \cdot \hat{e}^{(0)}\right\rangle=\int_{\omega} d^{3} \mathbf{x} \beta(\mathbf{x}, t) \hat{e}^{(0)}(\mathbf{x}, \mathbf{v}(t))
$$

The last term contains the history of the classical state parameters for $\tau \in[T, t]$ :

$$
\begin{aligned}
& \hat{S}_{t}[\beta(\tau), \mu(\tau), \mathbf{v}(\tau)]= \\
& \quad=\int_{\omega} d^{3} \mathbf{x}\left(\frac{\partial \beta(\mathbf{x}, \tau)}{\partial \tau} \hat{e}(\mathbf{x}, \tau-t)-\nabla \beta(\mathbf{x}, \tau) \cdot \hat{\mathbf{J}}_{e}(\mathbf{x}, \tau-t)\right)+ \\
& \quad+\int_{\partial \omega} d \sigma \mathbf{n} \beta(\mathbf{x}, \tau) \hat{\mathbf{J}}_{e}(\mathbf{x}, \tau-t)+\cdots
\end{aligned}
$$

where $\hat{\mathbf{J}}_{e}$ is the energy current:

$$
\frac{i}{\hbar}[\hat{H}, \hat{e}(\mathbf{x})]=-\operatorname{div} \hat{\mathbf{J}}_{\mathrm{e}}(\mathbf{x})
$$

the time dependence of the operators, e.g.: $\hat{e}(\mathbf{x}, t)$ means time dependence in Heisenberg picture: $\hat{e}(\mathbf{x}, t)=e^{\frac{i}{\hbar} \hat{H}} \hat{e}(\mathbf{x}) e^{-\frac{i}{\hbar} \hat{H} t}$; at the r.h.s. of equation (2.16) similar terms related to the other corresponding densities $\hat{m}(\mathbf{x}), \mathbf{p}(\mathbf{x})$ have been omitted for brevity. In the framework of information thermodynamics expression (2.15) would already be taken as a reliable description of the system: no wonder at all about the different structure of $\hat{\rho}_{T}$ and $\hat{\rho}_{t}$ since at time $t$ information on the history interval $[T, t]$ is available, while it was not for $t<T$. In our philosophy instead, $\hat{\rho}_{t}$ is an objective representation of the preparation of the system until time $t$; expression (2.15) indicates that the history can be relevant, so the choice (2.14) becomes highly critical: if the history of the system for $t<T$ is relevant, as one can expect looking at expression (2.15), the choice (2.14) (which is the most unbiased 
by the previous history) is wrong and also $\hat{\rho}_{t}$ given by (2.15) is meaningless. A way out could be to shift $T \rightarrow-\infty$, thus eliminating the previous history: however the infinite system limit must be taken before, just the contrary of our attitude; furthermore the classical parameters for remote times are not a practically available input; in this way, associating to $\hat{S}_{t}[\cdots]$ in $(2.15)$ a factor $e^{(\tau-t) \epsilon}$ and taking $T=-\infty$ one obtains Zubarev's non-equilibrium statistical operator (Zubarev 1974).

We propose another solution to the question whether (2.15) makes sense: by the very definition of the classical parameters, the history term in (2.15) is by construction irrelevant to calculate the expectations of the basic quantities $\hat{e}, \hat{m}, \hat{f}$ and $\hat{\mathbf{p}}$; one can expect that its contributions to the expectations of the corresponding time derivatives, e.g.:

$$
\dot{\hat{e}}=\frac{i}{\hbar}[\hat{H}, \hat{e}]=-\operatorname{div} \hat{\mathbf{J}}_{\mathrm{e}}
$$

is small enough to allow a perturbative expansion of the exponential in (2.15) with respect to the history term; then the classical parameters at time $\tau$ contribute to correlation functions of the type flow-flow or flow-density, e.g.: $\left\langle\hat{\mathbf{J}}_{e}(\mathbf{x}), \hat{e}(\mathbf{y}, \tau-t)\right\rangle$.

The short-time behavior of such correlation functions is rapidly decaying, when the time separation of the two functions becomes of the order of a suitable decay time $\tau_{c}$. Therefore if one considers $t>\tau_{c}$ only the part of the history referring to times $\tau>$ $t-\tau_{c}$ does appreciably contribute to the dynamics of the relevant variables. Then to compute their dynamical behavior for times $t, t-T>\tau_{c}$, the initial condition (2.14) is indeed the appropriate choice and consequently $\hat{\rho}_{t}$ given by (2.15) can be used to calculate the expectations of the time derivatives, thus yielding closed integrodifferential evolution equations for the classical variables

$$
\begin{gathered}
z(t) \equiv(\beta(t), \mu(t), \mathbf{v}(t)), \quad t \geq T+\tau_{c} \\
\operatorname{Tr}\left(\frac{\mathrm{i}}{\hbar}[\hat{\mathrm{H}}, \hat{\mathrm{e}}(\mathbf{x})] \hat{\rho}_{\mathrm{t}}\right)=\frac{\mathrm{d}}{\mathrm{dt}} \operatorname{Tr}(\hat{\mathrm{e}}(\mathbf{x}) \hat{\mathrm{w}}(\beta(\mathrm{t}), \mu(\mathrm{t}), \mathbf{v}(\mathrm{t}))) \\
\operatorname{Tr}\left(\frac{\mathrm{i}}{\hbar}[\hat{\mathrm{H}}, \hat{\mathrm{m}}(\mathbf{x})] \hat{\rho}_{\mathrm{t}}\right)=\frac{\mathrm{d}}{\mathrm{dt}} \operatorname{Tr}(\hat{\mathrm{m}}(\mathbf{x}) \hat{\mathrm{w}}(\beta(\mathrm{t}), \mu(\mathrm{t}), \mathbf{v}(\mathrm{t}))) \\
\operatorname{Tr}\left(\frac{\mathrm{i}}{\hbar}[\hat{\mathrm{H}}, \hat{\mathbf{p}}(\mathbf{x})] \hat{\rho}_{\mathrm{t}}\right)=\frac{\mathrm{i}}{\hbar} \operatorname{Tr}(\hat{\mathbf{p}}(\mathbf{x}) \hat{\mathrm{w}}(\beta(\mathrm{t}), \mu(\mathrm{t}), \mathbf{v}(\mathrm{t})))= \\
=\frac{d}{d t}(\mathbf{v}(\mathbf{x}, t) \operatorname{Tr}(\hat{\mathrm{m}}(\mathbf{x}) \hat{\mathrm{w}}(\beta(\mathrm{t}), \mu(\mathrm{t}), \mathbf{v}(\mathrm{t}))))
\end{gathered}
$$

With respect to these equations, the values of the state variables $z(t)$ within the time interval $\left[T, T+\tau_{c}\right]$ are prescribed parameters, related to the expectations of $\hat{e}(\mathbf{x}), \hat{m}(\mathbf{x})$ and $\mathbf{p}(\mathbf{x})$ : these expectations have the role of an input for the dynamics at times $t>T+\tau_{c}$. The time interval $\left[T, T+\tau_{c}\right]$ will be called preparation time interval of the system; during such time interval one might also assume that, due to the transition from $\hat{\psi}_{n c}$ to $\hat{\psi}$, the 
flows indicated in equation (2.16) do not vanish on the boundary $\partial \omega$, so that a surface contribution can arise. Neglecting mathematical problems about the existence of the solution of these evolution equations, it seems at first that one has solved in principle the question of a classical characterization of the finite isolated system: the parameters $z(t)$ establish the mathematical structure of the statistical operator that provides for times $t>T+\tau_{c}$ the expectations of the relevant observables. However a serious flaw is evident at long times: due to the pure point spectrum of $\hat{H}$ for a confined system, correlation functions have a quasiperiodical behavior, so they cannot decay indefinitely and as soon as memory is recovered, choice (2.14) is no longer tenable and then also $\hat{\rho}_{t}$ looses its meaning. Practically the difficulty can be avoided if one approximates expression (2.15), replacing the integral $\int_{T}^{t} d \tau \cdots$ by $\int_{t-\tau_{c}}^{t} d \tau \cdots$ and reproposing at time $t-\tau_{c}$ the initial condition

$$
\hat{\rho}_{t-\tau_{c}} \equiv \hat{w}\left[\beta\left(t-\tau_{c}\right), \mu\left(t-\tau_{c}\right), \mathbf{v}\left(t-\tau_{c}\right)\right]
$$

but this is to resort to an expedient. However it is the basic assumption of unitary dynamics for the isolated system that leads to this difficulty and just this assumption becomes questionable inside the framework which sets isolated systems as basic elements of reality.

\section{An opening to irreversibility}

As it is clear from Section 2 the preparation of a finite system is described by a statistical operator, in our aim bearing the classical state parameters of the system; instead the quantum state vector $\psi \in \mathcal{H}$, bearing indexes related to measurements of observables is a very strong idealization which applies to highly controlled preparations typical of particle physics. If preparations are represented by statistical operators, i.e. elements of the set $\mathcal{K}$, the base of the positive cone in the space $T(\mathcal{H})$ of trace-class operators in $\mathcal{H}$, it becomes most natural to describe transformations of preparations by positive trace-preserving maps $\mathcal{A}$ on $T(\mathcal{H})$, these maps taking now the role that unitary transformations play in the theory based on microsystems. If such a map $\mathcal{A}$ has an inverse one can show that $\mathcal{A} \cdot=\hat{U} \cdot \hat{U}^{\dagger}$ with $\hat{U}$ unitary or antiunitary on $\mathcal{H}$ and in this way the two formulations become equivalent; but $\mathcal{A}$ need not have an inverse. Denoting by $\hat{\rho}_{t}$ a preparation performed until time $t$, let us consider for a system isolated during the time interval $\left[t_{0}, t_{1}\right]$, the family of spontaneous repreparations $\rho_{t}, t \in\left[t_{0}, t_{1}\right]$, which arise due to the time evolution. One assumes that two preparations $\hat{\rho}_{t^{\prime}}, \hat{\rho}_{t^{\prime \prime}}, t_{0} \leq t^{\prime} \leq t^{\prime \prime} \leq t_{1}$ are connected to each other by a map $\mathcal{A}\left(t^{\prime \prime}-t^{\prime}\right)$ :

$$
\hat{\rho}_{t^{\prime \prime}}=\mathcal{A}\left(t^{\prime \prime}-t^{\prime}\right) \hat{\rho}_{t^{\prime}}
$$

the family $\mathcal{A}(\tau), \tau \geq 0$ being a semigroup of positive, trace-preserving maps. Actually taking the construction of section 2 into account, since one restricts to the relevant densities $\hat{e}(\mathbf{x}), \hat{\mathbf{p}}(\mathbf{x}), \hat{m}(\mathbf{x})(\hat{f}(\mathbf{x}, \mathbf{p}))$, looking at the time evolution in the Heisenberg picture, 
the properties of $\mathcal{A}^{\prime}(\tau)$, which are mappings on $\mathcal{B}(\mathcal{H})$ into $\mathcal{B}(\mathcal{H})$, are important and one could assume only that $\mathcal{A}^{\prime}(\tau)$ maps positive densities into positive operators and conserves basic constants of motion like mass and energy: $\mathcal{A}^{\prime}(\tau) \hat{M}=\hat{M}, \mathcal{A}^{\prime}(\tau) \hat{H}=\hat{H}$. Thus the basic feature of the description characterized by systems first and particles afterwards is irreversibility. In a sense we are now exploiting the arrow of time that is implicitly contained in the operative approach to QM, based on preparations and measurements of prepared systems(Bohm, 1993). On the other hand this almost trivial insertion of irreversibility into the formalism of QM can easily be pushed back, as it is shown in Ludwig's approach to QM of microsystems: in his approach a statistical operator represents an equivalence class of preparation procedures of a microsystem and an effect operator $\hat{F}, \quad(0 \leq \hat{F} \leq \hat{1})$ represents an equivalence class of registration procedures; any time shift of these procedures is allowed and still one assumes that for any preparation procedure another one, shifted back in time, can be found, equivalent to it. Then one arrives at a unitary representation of time shifts (Comi et al., 1975) and as a consequence, at unitary time evolution generated by the Hamiltonian; then also the fundamental principle of conservation of energy is most directly settled. The strategy we are proposing is to start always with local universal microphysics related to unitary representations of the fundamental symmetries, i.e. one has reversibility, energy conservation and some model leading to an energy density; e.g. in this preliminary discussion: expression (2.1).

Then, as we did in Section 2, one turns to the description of a system, characterized by a suitable choice of relevant variables. Let us specialize our macroscopic system to the case of a dilute gas with short range interaction $V(|\mathbf{r}|)$; it is well known that for such a system a Boltzmann type of description is satisfactory: this description is characterized by a typical macroscopic variation time $\tau_{1}$ much larger than the duration of a collision: the mean free path is much larger than the range of $V(|\mathbf{r}|)$. We shall see in Section 3 that the introduction of the time scale $\tau_{1}$, i.e.: the detailed dynamics of the two-body interaction is replaced by collision, leads from the energy density (2.1) to a semigroup $\mathcal{A}^{\prime}(\tau)$ of maps, that display a stronger form of positivity, called complete positivity which is reminiscent of the unitarity of the dynamics we started with; however this positivity will be relative to the relevant variables. We expect that the more general description based on equations (2.17) can be settled starting with a more fundamental expression than (2.1). Here $V(|\mathbf{x}|)$ is a phenomenological input that could be derived from a Hamiltonian describing the structure of the molecule as a bound state of charged particles: i.e. the very presence of $V(|\mathbf{r}|)$ indicates that more fundamental fields should be considered. When dealing with the Hamiltonian dynamics of the charged fields one introduces a time scale typical of center of mass motion of the bound states, one expects that a semigroup $\mathcal{A}^{\prime}(\tau)$ can be derived, whose generator displays an irreversible contribution together with the Hamiltonian contributions like (2.1). In this way the difficulty we met in Section 2 with the long time behavior of the correlation function, should eventually disappear. 


\section{Introduction of time scale and scattering map}

The relevant variables of the hydrodynamic or kinetic description have the following general structure, cf. Section 2:

$$
\sum_{h k} \hat{a}_{h}^{\dagger} A_{h k}(\xi) \hat{a}_{k}, \sum_{k_{1} k_{2} h_{1} h_{2}} \hat{a}_{h_{1}}^{\dagger} \hat{a}_{h_{2}}^{\dagger} A_{h_{1} h_{2} k_{2} k_{1}}(\mathbf{x}) \hat{a}_{k_{2}} \hat{a}_{k_{1}}
$$

thus, in Heisenberg picture, we have to study expressions of the form:

$$
\sum_{h k} e^{\frac{i}{\hbar} \hat{H} t} \hat{a}_{h}^{\dagger} \hat{a}_{k} e^{-\frac{i}{\hbar} \hat{H} t} A_{h k}(\xi) \quad \sum_{k_{1} k_{2} h_{1} h_{2}} e^{\frac{i}{\hbar} \hat{H} t} \hat{a}_{h_{1}}^{\dagger} \hat{a}_{h_{2}}^{\dagger} \hat{a}_{k_{2}} \hat{a}_{k_{1}} e^{-\frac{i}{\hbar} \hat{H} t} A_{h_{1} h_{2} k_{2} k_{1}}(\mathbf{x})
$$

where restriction to slow variables means that in the sums (4.1) only terms are considered such that:

$$
\frac{1}{\hbar}\left|E_{h}-E_{k}\right|<\frac{1}{\tau_{1}} \quad \frac{1}{\hbar}\left|E_{h_{1}}+E_{h_{2}}-E_{k_{1}}-E_{k_{2}}\right|<\frac{1}{\tau_{1}}
$$

where $\tau_{1}$ is the typical variation time of the Boltzmann description: $\tau_{1} \sim 10^{-13} \mathrm{sec}$, the time interval between two collisions. The Hamiltonian $\hat{H}$, given by (2.13) generates an isomorphism $\mathcal{U}_{H}^{\prime}(t)$ of $\mathcal{B}(\mathcal{H})$ :

$$
\mathcal{U}_{H}^{\prime}(t) \cdot=e^{\frac{i}{\hbar} \hat{H} t} \cdot e^{-\frac{i}{\hbar} \hat{H} t}=\int_{-i \infty+\epsilon}^{i \infty+\epsilon} d z \frac{e^{z t}}{2 \pi i} \frac{1}{z-\mathcal{H}^{\prime}}
$$

where $\mathcal{H}^{\prime} \cdot=\frac{i}{\hbar}[\hat{H}, \cdot]$. We shall introduce a formalism reminiscent of usual scattering theory shifting the space $\mathcal{H}$ to $\mathcal{B}(\mathcal{H})$ and operators in $\mathcal{H}$ to maps in $\mathcal{B}(\mathcal{H})$; for brevity only the main steps of the treatment are indicated:

$$
\begin{gathered}
\frac{1}{z-\mathcal{H}^{\prime}}=\frac{1}{z-\mathcal{H}_{0}^{\prime}}+\frac{1}{z-\mathcal{H}_{0}^{\prime}} \mathcal{T}(z) \frac{1}{z-\mathcal{H}_{0}^{\prime}} \\
\mathcal{T}(z) \equiv \mathcal{V}^{\prime}+\mathcal{V}^{\prime} \frac{1}{z-\mathcal{H}^{\prime}} \mathcal{V}^{\prime}
\end{gathered}
$$

where $\mathcal{H}_{0}^{\prime}=\frac{i}{\hbar}\left[\hat{H}_{0}, \cdot\right], \mathcal{V}^{\prime}=\frac{i}{\hbar}\left[\hat{H}-\hat{H}_{0}, \cdot\right], \hat{H}_{0}=\sum_{f} E_{f} \hat{a}_{f}^{\dagger} \hat{a}_{f}$. The operators

$$
\hat{a}_{h_{1}}^{\dagger} \hat{a}_{h_{2}}^{\dagger} \cdots \hat{a}_{h_{r}}^{\dagger} \hat{a}_{k_{s}} \cdots \hat{a}_{k_{2}} \hat{a}_{k_{1}}
$$

are eigenstates of $\mathcal{H}_{0}^{\prime}$ with eigenvalues $\frac{i}{\hbar}\left(E_{h_{1}}+E_{h_{2}} \cdots+E_{h_{r}}-E_{k_{1}}-E_{k_{2}}-\cdots-E_{k_{s}}\right)$. By the basic algebraic property:

$$
\mathcal{U}^{\prime}(t) \hat{a}_{h}^{\dagger} \hat{a}_{k}=\left(\mathcal{U}^{\prime}(t) \hat{a}_{h}\right)^{\dagger}\left(\mathcal{U}^{\prime}(t) \hat{a}_{k}\right),
$$

it is clear that the main formal tool to treat expressions 4.1 is the representation of the operator $\mathcal{T}(z) \hat{a}_{k}$, in terms of the basis 4.6. By conservation of total mass one has the general structure:

$$
\begin{aligned}
\mathcal{T}(z) \hat{a}_{k}= & \sum_{f} A_{k f}(z, \hat{n}) \hat{a}_{f}+\sum_{l f_{2} f_{1}} \hat{a}_{l}^{\dagger} A_{l k f_{2} f_{1}}(z, \hat{n}) \hat{a}_{f_{2}} \hat{a}_{f_{1}}+ \\
& +\sum_{l_{1} l_{2} f_{3} f_{2} f_{1}} \hat{a}_{l_{1}}^{\dagger} \hat{a}_{l_{2}}^{\dagger} A_{l_{1} l_{2} k f_{3} f_{2} f_{1}}(z, \hat{n}) \hat{a}_{f_{3}} \hat{a}_{f_{2}} \hat{a}_{f_{1}}+\cdots
\end{aligned}
$$


where the coefficients $A_{l k f_{2} f_{1}}(z, \hat{n}), A_{l_{1} l_{2} k f_{3} f_{2} f_{1}}(z, \hat{n})$ are operator functions of the set of number operators $\hat{n}_{h}=\hat{a}_{h}^{\dagger} \hat{a}_{h}$, i.e.: they are diagonal with respect to the basis in Fock space, generated by the creation operators. A very natural approximation in usual kinetic theory is the evolution by two-particle collisions. In our field description the corresponding approximation seems to be the following: evolution involving only one other field mode; i.e. one would break up expansion (4.8) after the first two terms; however all the spectator modes are also relevant through the $\hat{n}$ dependence of the coefficients and provide the Pauli principle corrections. Then also the third term in (4.8) contributes to these Pauli principle corrections inside an expression of the form $\hat{a}_{h}^{\dagger} \mathcal{T}(z) \hat{a}_{k}$ when some index $f_{1}, f_{2}, f_{3}$ is equal to $h$. Let us indicate briefly the structure of the coefficient $A_{l k f_{1} f_{2}}(z, \hat{n})$ in (4.8); it is given essentially by the matrix elements of a two-particle scattering operator, bearing Pauli-principle corrections, defined as follows:

$$
\hat{T}^{(2)}(z)=\hat{V}^{(2)}+\hat{V}^{(2)} \frac{1}{z-\hat{H}_{L}^{(2)}} \hat{V}_{L}^{(2)} \quad, \quad \hat{H}_{L}^{(2)}=\hat{H}_{0}^{(2)}+\hat{V}_{L}^{(2)}
$$

the operators labeled by the index (2) are defined in the Hilbert space $\mathcal{H}^{(2)}$ of two identical particles by matrix elements in the two particle (symmetric or antisymmetric) basis $\mid l_{1} l_{2}>$ :

$$
\begin{array}{r}
<l_{2} l_{1}\left|\hat{H}_{o}^{(2)}\right| f_{1} f_{2}>=\left(E_{f_{1}}+E_{f_{2}}\right) \frac{1}{2 !}\left(\delta_{l_{2} f_{2}} \delta_{l_{1} f_{1}} \pm \delta_{l_{2} f_{1}} \delta_{l_{1} f_{2}}\right) \\
<l_{2} l_{1}\left|\hat{V}^{(2)}\right| f_{1} f_{2}>=V_{l_{1} l_{2} f_{2} f_{1}} \\
<l_{2} l_{1}\left|\hat{V}_{L}^{(2)}\right| f_{1} f_{2}>=\left(1 \pm \hat{n}_{l_{1}} \pm \hat{n}_{l_{2}}\right) V_{l_{1} l_{2} f_{2} f_{1}}
\end{array}
$$

These two-particle quantum mechanical elements, are produced in a natural way by the quantum field structure and are constructed with the coefficients $E_{f}, V_{l_{1} l_{2} f_{2} f_{1}}$, arising in the Hamiltonian (2.13), written in terms of $\hat{a}_{l}^{\dagger}, \hat{a}_{f}$ :

$$
\hat{H}=\sum_{f} E_{f} \hat{a}_{f}^{\dagger} \hat{a}_{f}^{\dagger}+\frac{1}{2} \sum_{l_{1} l_{2} f_{1} f_{2}} \hat{a}_{l_{1}}^{\dagger} \hat{a}_{l_{2}}^{\dagger} V_{l_{1} l_{2} f_{2} f_{1}} \hat{a}_{f_{2}} \hat{a}_{f_{1}}
$$

The factor $\left(1 \pm \hat{n}_{l_{1}} \pm \hat{n}_{l_{2}}\right)$ in the last of equations ( 4.10$)$ represents the Pauli-principle correction, it is an operator valued expression, but this makes no problem for the definition (4.9) of $\hat{T}^{(2)}(z)$ since for all $l_{1}, l_{2}$ they commute. We assume for simplicity that no bound states between the molecules can be formed, this means that in the thermodynamic limit the coefficients in (4.8) have no singularities on the imaginary axis. Then the time scale is introduced by the following modifications. First the expression $\mathcal{T}(z) \hat{a}_{k}$ is replaced by $\mathcal{T}(z+\eta) \hat{a}_{k}$, with $\eta \approx \frac{\hbar}{\tau_{0}}, \tau_{0}$ being of the order of the collision time. Final results for expectations of the relevant variables, having a typical variation time $\tau_{1} \gg \tau_{0}=\frac{\hbar}{\eta}$ are practically independent on $\eta$ : actually only these $\eta$ independent results are significant in our essentially incomplete description of the finite system; $\eta$ dependence would mean dependence on the distribution of the huge set of poles that $\mathcal{T}(z)$ has on the imaginary 
axis, which in turn is related to the confinement of the system, only roughly represented by the boundary conditions we assumed in Section 2; so $\eta$ dependence is more an artifact of the idealized confinement than a physical feature. A second change concerns the external variables $E_{h}, E_{k}$; we set for them $E_{h}=E_{h k}+\frac{1}{2} \xi_{h k}, E_{k}=E_{h k}-\frac{1}{2} \xi_{h k}$ and the replace $\xi_{h k} \rightarrow \xi_{h k}-2 i \epsilon$, with $\eta>\epsilon>>\frac{\hbar}{\tau_{1}}$, thus implying some smoothness property of the dependence on the variable $\xi_{h k}$, as the existence of an analytic continuation into the lower half-plane. Now expressions (4.7) are calculated taking into account equations (4.3), (4.4) and the representation (4.8) where $z \rightarrow z+\eta$. Then one can separate in the calculation of (4.3) the contribution of the singularities of $\frac{1}{z-\mathcal{H}_{0}^{\prime}}$ from the singularities at points $z$, with Rez $\leq-\eta$ and neglect the last ones: their contribution is negligible for $t>>\tau_{0}$ if we consider only relevant variables. Finally one arrives with some calculations to the following very perspicuous representation of expression (4.7):

$$
\mathcal{U}^{\prime}(t) \hat{a}_{h}^{\dagger} \hat{a}_{k}=\hat{a}_{h}^{\dagger} \hat{a}_{k}+t \mathcal{L}^{\prime}\left(\hat{a}_{h}^{\dagger} \hat{a}_{k}\right) \quad t>>\tau_{o}, \quad t \sim \tau_{1}
$$

where

$$
\mathcal{L}^{\prime} \hat{a}_{h}^{\dagger} \hat{a}_{k}=\frac{i}{\hbar}\left[\hat{H}_{\mathrm{eff}}, \hat{a}_{h}^{\dagger} \hat{a}_{k}\right]-\frac{1}{\hbar}\left(\left[\hat{\Gamma}, \hat{a}_{h}^{\dagger}\right] \hat{a}_{k}-\frac{1}{\hbar} \hat{a}_{h}^{\dagger}\left[\hat{\Gamma}, \hat{a}_{k}\right]\right)+\frac{1}{\hbar} \sum_{\lambda} \hat{R}_{h \lambda}^{\dagger} \hat{R}_{k \lambda}
$$

In the first term at the r.h.s. of equation (4.12) an effective Hamiltonian appears, given by equation (4.11) with $V_{l_{1} l_{2} f_{2} f_{1}}$ replaced by $V_{l_{1} l_{2} f_{2} f_{1}}^{\text {eff }}$,

$$
V_{l_{1} l_{2} f_{2} f_{1}}^{\mathrm{eff}}==<l_{2} l_{1}\left|\frac{1}{2}\left(\hat{T}^{(2)}\left(E_{f_{1}}+E_{f_{2}}+i \eta \hbar\right)+\hat{T}^{(2)}\left(E_{l_{1}}+E_{l_{2}}+i \eta \hbar\right)^{\dagger}\right)\right| f_{2} f_{1}>
$$

Thus the interaction potential is replaced by the self-adjoint part of the scattering operator; the remaining part of the scattering operator is not zero if one goes beyond the Born approximation and yields the second term at r.h.s. of equation (4.12), which is no longer of the form $\left[\cdot, \hat{a}_{h}^{\dagger} \hat{a}_{k}\right]$,

$\hat{\Gamma}=\frac{1}{2} \sum_{f_{1} f_{2} l_{1} l_{2}} \hat{a}_{l_{1}}^{\dagger} \hat{a}_{l_{2}}^{\dagger}<l_{2} l_{1}\left|\frac{i}{2}\left(\hat{T}^{(2)}\left(E_{f_{1}}+E_{f_{2}}+i \eta \hbar\right)-\hat{T}^{(2)}\left(E_{l_{1}}+E_{l_{2}}+i \eta \hbar\right)^{\dagger}\right)\right| f_{2} f_{1}>\hat{a}_{f_{2}} \hat{a}_{f_{1}}$

The operators $\hat{R}_{k \lambda}$ are given by:

$$
\hat{R}_{k \lambda}=-i \sqrt{2 \epsilon\left(1 \pm \hat{n}_{\lambda} \pm \hat{n}_{k}\right)} \sum_{f_{1} f_{2}} \frac{<k \lambda \mid \hat{T}^{(2)}\left(E_{f_{1}}+E_{f_{2}}+i \hbar(\eta-\epsilon)\right)}{\left.E_{k}+E_{\lambda}-E_{f_{1}}-E_{f_{2}}\right)-i \hbar \epsilon} \hat{a}_{f_{2}} \hat{a}_{f_{1}},
$$

the factor $\sqrt{2 \epsilon\left(1 \pm \hat{n}_{\lambda} \pm \hat{n}_{k}\right)}$ arising by the approximation:

$$
2 \epsilon\left(1 \pm \hat{n}_{\lambda} \pm\left(\hat{n}_{k} \pm \hat{n}_{k}\right)\right) \approx \sqrt{2 \epsilon\left(1 \pm \hat{n}_{\lambda} \pm \hat{n}_{h}\right)} \sqrt{2 \epsilon\left(1 \pm \hat{n}_{\lambda} \pm \hat{n}_{k}\right)}
$$


which holds in the case of not too large Pauli principle corrections. Because of mass conservation one has within our approximations:

$$
\hat{\Gamma}^{(2)} \approx \frac{1}{4} \sum_{h \lambda} \hat{R}_{h \lambda}^{\dagger} \hat{R}_{k \lambda}
$$

or in order to have exactly $\mathcal{L}^{\prime} \hat{M}=0$, one can set in equation (4.12):

$$
\hat{\Gamma}^{(2)}=\frac{1}{4} \sum_{h \lambda} \hat{R}_{h \lambda}^{\dagger} \hat{R}_{h \lambda}
$$

Let us notice that the general structure of $\mathcal{L}^{\prime}$, arising from the factorized form shown by (4.7), indicates a form of complete positivity relative to the operators $\hat{a}_{h}^{\dagger} \hat{a}_{k}$; one can easily see that:

$$
0 \leq \sum_{h k}<\psi_{h} \mid\left(\left[1+t \cdot \mathcal{L}^{\prime}\right) \hat{a}_{h}^{\dagger} \hat{a}_{k} \mid \psi_{k}>\quad \forall \quad\left\{\psi_{h}\right\} \subset \mathcal{H}\right.
$$

to first order in $\tau$. Assuming (2.1) as fundamental energy density, introducing confinement and a time scale much larger than $\tau_{0}$, we arrived by a systematic procedure to the generator $\mathcal{L}^{\prime}$ : the effective Hamiltonian is now associated with a non Hamiltonian contribution. By a similar treatment for the system consisting of a particle interacting with a medium, one can obtain $\mathcal{L}^{\prime}$ for the particle variables (Lanz, Vacchini, 1997), then considering the time evolution of the statistical operator for the sole particle, the typical quantum master equation describing Brownian motion is obtained.

In our present case we can expect that, on the time scale ruled by $\mathcal{L}^{\prime}$, no memory of the classical state variables introduced in Section 2 is relevant, so that one has a closed set of evolution equations:

$$
\operatorname{Tr}\left(\left(\mathcal{L}^{\prime} \hat{\mathrm{f}}(\mathbf{x})\right) \hat{\mathrm{w}}(\beta(\mathrm{t}), \mu(\mathrm{t}), \mathbf{v}(\mathrm{t}))=\frac{\mathrm{d}}{\mathrm{dt}} \operatorname{Tr}(\hat{\mathrm{f}}(\mathbf{x}) \hat{\mathrm{w}}(\beta(\mathrm{t}), \mu(\mathrm{t}) \mathbf{v}(\mathrm{t}))\right.
$$

where $\hat{f}(\mathbf{x})$ are the relevant fields $\hat{e}(\mathbf{x}), \mathbf{p}(\mathbf{x}), \hat{m}(\mathbf{x})$. Looking at $\mathcal{L}^{\prime}\left(\hat{a}_{h}^{\dagger} \hat{a}_{h}\right)$ one can see that $\sum \hat{R}_{h \lambda}^{\dagger} \hat{R}_{h \lambda}$ has the typical structure of the gain contribution by a collision ending up in the two-particle state $h \lambda$, which is present in the Boltzmann collision term, while $-\frac{1}{\hbar}\left(\left[\Gamma, \hat{a}_{h}^{\dagger}\right] \hat{a}_{h}-\hat{a}_{h}^{\dagger}\left[\hat{\Gamma}, \hat{a}_{h}\right]\right)$ yields the loss term by a collision involving a particle in the state $h$. Therefore one can expect that the description based on equations (4.15) is an improvement of the usual Boltzmann equation, because no factorization hypothesis of two-particle distribution function must be used and $\hat{H}^{\text {eff }}$ is not purely kinetic. We hope that the procedure used to obtain $\mathcal{L}^{\prime}$, which is based on smoothness properties of the scattering map $\mathcal{T}(z)$ can be extended to the case of singularities of $\mathcal{T}(z)$ related to bound states: so one could also make that the energy density (2.1) from which we started, fits in a suitable $\mathcal{L}^{\prime}$, derived by a more fundamental model. 


\section{References}

Bohm, A. (1993). Gamow vectors and the arrow of time, in Symposium On the Foundations of Modern Physics, (1993) Busch, P., Lahti, P. J., and Mittelstaedt, P., eds., World Scientific, Singapore, p.77-97

Comi, M., Lanz, L., Lugiato, L.A., and Ramella, G., (1975) Journ. Math. Phys. 16, 910 Davies, E. B. (1976). Quantum Theory of Open Systems, Academic Press, London.

Holevo, A. S. (1982). Probabilistic and Statistical Aspects of Quantum Theory, North Holland, Amsterdam.

Kraus, K. (1983). States, Effects and Operations, in Lecture Notes in Physics, Volume 190, Springer, Berlin.

Lanz, L., Melsheimer, O. and Wacker, E. (1985) Physica, 131A, 520

Lanz, L., and Vacchini, B. (1997). International Journal of Theoretical Physics, 36, 67.

Ludwig, G. (1983). Foundations of Quantum Mechanics, Springer, Berlin.

Robin, W. A. (1990). Journal of Physics A, 23, 2065.

Zubarev, D. N. (1974). Non-equilibrium statistical thermodynamics, Consultant Bureau, New York. 